\title{
Non-paralytic motor disturbances and speech disorders: the role of the supplementary motor area
}

\author{
HJ GELMERS
}

\author{
From the Department of Neurology, Pr Irene Ziekenhuis, Almelo, The Netherlands
}

SUMMARY A right-handed patient with a lesion demonstrated by CT to involve the right medial frontal cortex is described. He exhibited a strong contralateral grasp reflex, motor perseveration and the presence of purposeful movements that appeared to be dissociated from conscious volition. In addition, there was a disorder of speech consisting of a lack of spontanteous speech production, with preserved ability to imitate. It is suggested that these disorders are due to damage to the supplementary motor area.

Experimental studies in man ${ }^{1}$ indicate that there are a number of cortical fields involved in motor control, among which is the supplementary motor area. Although its functional distinctiveness has been demonstrated, there are few descriptions of the results of supplementary motor area damage..$^{351213}$ The purpose of this case history is to describe the clinical symptoms caused by a lesion involving the right medial frontal cortex and to outline the "supplementary motor area-syndrome".

\section{Case history}

A 69-year-old right-handed man had sudden onset of difficulty in speaking, together with weakness of the left leg. Examination on admission showed him to be awake, alert and oriented. He had a mild left-sided lower facial weakness at rest and on volition, with a striking weakness after laughing. There was total paralysis of the left leg. Although the left arm showed a global inertia and no spontaneous movements, there was no paresis. The patient found it an immense effort to move. When he moved, the movements were correct but slow and deliberate. A strong grasp reflex was noted in the left hand. Tendon reflexes were exaggerated on the left and plantar responses were extensor on the left and equivocal on the right. Visual fields and ocular movements were full. Pinprick and vibratory sensation was normal as were detection of passive

Address for reprint requests: Dr HJ Gelmers, Department of Neurology, Pr Irene Ziekenhuis, 7607 PW Almelo, The Netherlands.

Received 15 December 1982 and in revised form 22 May 1983. Accepted 2 June 1983 movements and identification of palpated objects. The patient did not initiate conversation. He spoke very haltingly in reply to questions. The responses were characterised by a great deal of difficulty in initiation. Comprehension of speech was good, as was the ability to imitate complex phrases sentences. There were no localising phenomena indicating parietal lobe damage; the interpretation of body-perception was normal. Computed tomograms demonstrated an area of increased density involving the right medial frontal cortex (2nd day after onset of symptoms) (fig). Ten days after onset there was still a strong grasp reflex in the left arm. The left leg had improved in strength, but remained weak. The patient was observed to reach out spontaneously with the left arm and then would not be able to release his grip voluntarily. He began to perseverate with his left arm when using a knife and fork during meals, or when handling a haircomb or an electrical razor. This motor perseveration was inhibited to some extent by concentrated effort. After five weeks the patient began to walk unaided, but he still displayed motor perseveration of the left arm. This phenomenon was indeed perseveration and not tonic innervation or an inability to release objects on account of the grasp reflex.

\section{Discussion}

This patient displayed a strong grasp reflex of the left hand initially preceded by a global inertia. He was observed to have motor perseveration (inappropriate repetition of a simple movement) of the left hand. Later it was noted that apparently purposeful movements appeared to be occurring independent of conscious volitional control. Additionally, the patient exhibited a speech disorder, consisting of a lack of spontaneous speech with preserved 


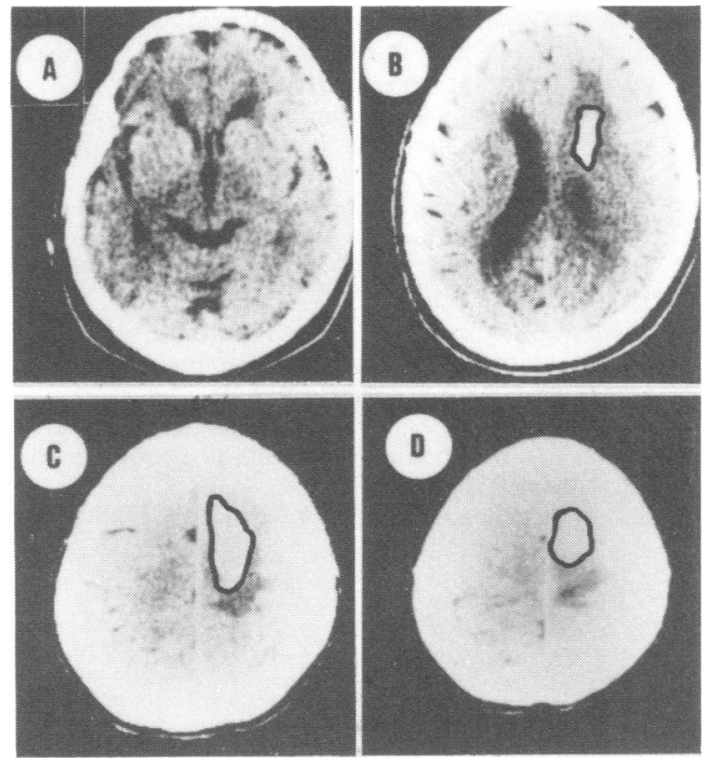

Fig Selection of slices from a complete set of CT-scans. The infraventricular (not depicted) and low ventricular $(A)$ slices were normal. The high ventricular slice $(B)$ demonstrated an area of increased density involving the medial frontal area of the right hemisphere. In the supraventricular slices $(C, D)$ the extension of the increased area is shown (the lesion is for reasons of clarity outlined in black ink).

ability to repeat. CT showed a lesion involving the right medial frontal cortex.

A patient with some similar features has been published, ${ }^{2}$ who suffered an infarction of the medial frontal cortex due to occlusion of the left pericallosal artery. This patient had spontaneous grasping together with motor perseveration of the right hand. $\mathrm{He}$ too was unable to inhibit these movements, although he was fully aware of them. The ability to execute new movements was retained. Others ${ }^{3}$ have described global akinesia of the right arm in patients with lesions involving the left medial frontal cortex. All these motor phenomena were observed in our patient. More widespread dissociative psychomotor phenomena, including the "alien hand sign" and "intermanual conflict" as observed in patients the first weeks following section of the corpus callosum for intractable epilepsy, ${ }^{4}$ were not seen in our patient. This is contrary to other observers ${ }^{5}$ who, in patients with medial frontal cortex infarction, noted that one of the hands behaved in a way which the patient found "alien" or at least uncooperative.

Two functional zones on the medial surface of the frontal lobes, damage to which may be implicated in the appearance of motor disturbance, as well as the speech disorder, are the supplementary motor area and the cingulate cortex. Penfield and Jasper ${ }^{1}$ reported that stimulation of the supplementary motor area produced vocalisation, speech arrest, complex coordinated or repetitive movements usually appearing in the contralateral arm and arrest or slowing of voluntary action following which it was noted that the patient was "usually puzzled by his inability to execute" the action he had intended. Continuous electrical stimulation of anterior cingulate cortex in epileptic patients is followed by complex, highly integrated forms of motor behaviour most often in the contralateral arm. ${ }^{6}$ Recently, regional blood flow studies in man have demonstrated bilateral activation of supplementary motor area in association with various complex movements of the extremities, ${ }^{78}$ as well as with speech. ${ }^{910}$ From these studies it has been suggested that the supplementary motor area is involved in the assembly and execution of time-ordered sequences of motor instructions. ${ }^{11}$

Our patient also exhibited a speech disorder, consisting of a lack of spontaneous speech production, with preserved ability to imitate. This type of speech disturbance is often designed as transcortical motor aphasia ${ }^{12}$ and has been described in patients who sustained damage to the left medial frontal cortex. ${ }^{512}{ }^{13}$ Although similar disturbances of speech of patients have been recorded by others, ${ }^{3}$ no mention was made of the site of the involving mediofrontal lesion. Since the lesion in our patients was localised in the right hemisphere, it is best not to describe this speech disorder as an "aphasia". Moreover, it covers the recently proposed term "transcortical motor aprosodia". ${ }^{14}$

There seems now sufficient clinical and experimental evidence to conclude that lesions of the medial frontal parts of the hemisphere, including the supplementary motor area, gives rise to nonparalytic motor and speech disturbances.

The author is grateful to Professor Dr J Willemse, Department of Child Neurology, Faculty of Medicine, University of Utrecht, for support and advice in this study.

\section{References}

${ }^{1}$ Penfield W, Jasper H. Epilepsy and the Functional Anatomy of the Human Brain. Boston: Little Brown, 1954:88-108.

${ }^{2}$ Shahani B, Burrows P, Whitty CWM. The grasp reflex and perseveration. Brain 1970;93:181-92.

${ }^{3}$ Damasio AR, Hoesen GW van. Structure and function of the supplementary motor area. Neurology (Minneap) 1980;30:359. 
${ }^{4}$ Bogen JE. The callosal syndrome. In: Heilman KM, Valenstein E, eds. Clinical Neuropsychology. New York: New York University Press, 1979:308-59.

${ }^{5}$ Goldberg G, Mayer NH, Toglia JU. Medial frontal cortex infarction and the alien hand sign. Arch Neurol 1981;38:683-6.

${ }^{6}$ Talairach J, Bancaud J. The supplementary motor area in man: anatomo-functional findings by stereoelectroencephalography in epilepsy. Int $J$ Neurol 1966;5:330-47.

${ }^{7}$ Roland PE, Skinhøj E, Lassen NA, et al. Supplementary motor area and other cortical areas in organization of voluntary movements in man. $J$ Neurophysiol 1980 ; 43: $118-36$

${ }^{8}$ Gelmers HJ. Cortical organization of voluntary motor activity as revealed by measurements of regional cerebral blood flow. J Neurol Sci 1981;52:149-61.
${ }^{9}$ Larsen B, Skinhøj E, Lassen NA. Variations in regional cortical blood flow in the right and left hemispheres during automatic speech. Brain 1978;101:193-209.

${ }^{10}$ Gelmers HJ. Regional cerebral blood flow correlates of speech in the dominant and in the non-dominant hemisphere. J Neurol Sci (In press).

"Orgogozo JM, Larsen B. Activation of the supplementary motor area during voluntary movement in man suggests it works as a supra-motor area. Science 1979;206:847-50.

${ }^{12}$ Masdeu JC, Schoene WC, Funkenstein H. Aphasia following infarction of the left supplementary motor area. Neurology (Minneap) 1978;28:1220-3.

${ }^{13}$ Alexander MP, Schmidt MA. The aphasia syndrome of stroke in the left anterior cerebral artery territory. Arch Neurol 1980;37:97-100.

${ }^{14}$ Ross ED. The aprosodias. Arch Neurol 1981;38:561-9. 